\title{
SOLUTION OF HYBRID TIME FRACTIONAL DIFFUSION PROBLEM VIA WEIGHTED INNER PRODUCT
}

\author{
Suleyman Cetinkaya, Ali Demir \\ Faculty of Arts and Sciences, Kocaeli University \\ Kocaeli, Turkey \\ suleyman.cetinkaya@kocaeli.edu.tr,ademir@kocaeli.edu.tr
}

Received: 15 December 2020; Accepted: 10 June 2021

\begin{abstract}
In this research, we discuss the construction of the analytic solution of homogenous initial boundary value problem including partial differential equations of fractional order. Since the homogenous initial boundary value problem involves the Hybrid fractional order derivative with various coefficients functions, it has classical initial and boundary conditions. By means of separation of the variables method and the inner product defined on $L^{2}[0, l]$, the solution is constructed in the form of a Fourier series including the bivariate Mittag-Leffler function. An illustrative example presents the applicability and influence of the separation of variables method on time fractional diffusion problems. Moreover, as the fractional order $\alpha$ tends to 1 , the solution of the fractional diffusion problem tends to the solution of the diffusion problem which proves the accuracy of the solution.
\end{abstract}

MSC 2010: 26A33, $65 M 70$

Keywords: hybrid fractional derivative, bivariate Mittag-Leffler function, Dirichlet boundary conditions, spectral method, weighted inner product

\section{Introduction}

Since mathematical models including fractional derivatives play a vital role, fractional derivatives have drawn increasing attention from many researchers in various branches of science. Therefore, there are many different fractional derivatives such as Caputo, Riemann-Liouville, Atangana-Baleanu defined as follows: The $q^{\text {th }}$ order fractional derivative of $u(t)$ in Caputo sense is defined as

$$
D^{q} u(t)=\frac{1}{\Gamma(n-q)} \int_{t_{0}}^{t}(t-s)^{n-q-1} u^{(n)}(s) d s, t \in\left[t_{0}, t_{0}+T\right]
$$

where $u^{(n)}(t)=\frac{d^{n} u}{d t^{n}}, n-1<q<n$. 
The $q^{t h}$ order fractional derivative of $u(t)$ in the Riemann-Liouville sense is defined as

$$
D^{q} u(t)=\frac{1}{\Gamma(n-q)} \frac{d^{n}}{d t^{n}} \int_{t_{0}}^{t}(t-s)^{n-q-1} u(s) d s, t \in\left[t_{0}, t_{0}+T\right]
$$

where $n-1<q<n$.

However these fractional derivatives do not satisfy the most important properties of the ordinary derivative, which leads to many difficulties in analyzing or obtaining the solution of fractional mathematical models.

As a result, many scientists focus on defining new fractional derivatives to cover the setbacks of the defined ones. Moreover, the success of mathematical modelling of systems or processes depends on the fractional derivative it involves, since the correct choice of the fractional derivative allows us to model the real data of systems or processes accurately. In order to the define new fractional derivatives, various methods exist, and these are classified based on their features and formation such as nonlocal fractional derivatives and local fractional derivatives. The constant proportional Caputo hybrid operator is a newly defined fractional derivative that is a combination of the Caputo derivative and the proportional derivative and is defined as:

$$
\begin{aligned}
{ }_{0}^{C P C} D_{t}^{\alpha} f(t) & =\frac{1}{\Gamma(1-\alpha)} \int_{0}^{t}\left(K_{1}(\alpha) f(\tau)+K_{0}(\alpha) f^{\prime}(\tau)\right)(t-\tau)^{-\alpha} d \tau \\
& =K_{1}(\alpha){ }_{0}^{R L} I_{t}^{1-\alpha} f(t)+K_{0}(\alpha){ }_{0}^{C} D_{t}^{\alpha} f(t)
\end{aligned}
$$

where the functions $K_{0}$ and $K_{1}$ satisfy certain properties in terms of limit [1]. The domain of this operator contains functions $f$ on positive reals such that $f$ and its derivative $f^{\prime}$ are locally $L^{1}$ functions. Moreover, ${ }_{0}^{R L} I_{t}^{\alpha}$ and ${ }_{0}^{C} D_{t}^{\alpha}$ represent the Riemann-Liouville integral and Caputo derivative, respectively.Note that this hybrid fractional operator can be enounced as a linear combination of the Caputo fractional derivative and the Riemann-Liouville fractional integral. Notice that the constant proportional Caputo hybrid operator is obtained by adding a non-locality property to a proportional derivative operator, which allows us to model processes with non-local behaviour more efficiently and is the most important advantage of it. The non-locality property of the constant proportional Caputo hybrid operator is a result of the Riemann-Liouville integral which is defined as:

The Riemann-Liouville time-fractional integral of a real valued function $u(x, t)$ is defined as

$$
I_{t}^{\alpha} u(x, t)=\frac{1}{\Gamma(\alpha)} \int_{0}^{t}(t-s)^{\alpha-1} u(x, s) d s
$$

where $\alpha>0$ denotes the order of the integral.

This new fractional derivative is drawing the attention of many researchers in various branches of science and, as a result, there is a substantial amount of study in the literature such as on the hybrid fractional derivative [2-6], heat and mass transportation [7-10] and dynamics of processes [11-14]. 
The choice of functions $K_{0}$ and $K_{1}$ included in the definition of the constant proportional Caputo hybrid operator motivates us to analyze the solution of fractional diffusion equations with initial and boundary conditions for various functions $K_{0}$ and $K_{1}$ and compare them. In this study, we focus on obtaining the solution of the following fractional diffusion equation with various the constant proportional Caputo hybrid operator by making use of the separation of variables method:

$$
\begin{aligned}
{ }_{0}^{C P C} D_{t}^{\alpha} u(x, t) & =u_{x x}(x, t)+B u_{x}(x, t)-C u(x, t), \\
u(0, t) & =u(l, t)=0, \\
u(x, 0) & =f(x) e^{-\frac{B}{2} x},
\end{aligned}
$$

where $0<\alpha<1,0 \leq x \leq l, 0 \leq t \leq T, B, C \in \mathbb{R}$. Here we use the following forms of the proportional derivatives: We especially consider the following ones:

$$
\begin{aligned}
& { }_{0}^{C P C} D_{\alpha}{ }^{1} f(t)=(1-\alpha){ }_{0}^{R L} I_{t}^{1-\alpha} f(t)+\alpha_{0}^{C} D_{t}^{\alpha} f(t), \\
& { }_{0}^{C P C} D_{\alpha}{ }^{2} f(t)=\left(1-\alpha^{2}\right){ }_{0}^{R L} I_{t}^{1-\alpha} f(t)+\alpha_{0}^{2 C} D_{t}^{\alpha} f(t) .
\end{aligned}
$$

The novelty of this study is the application of the separation of variables method to a time fractional diffusion equation including the constant proportional Caputo hybrid operator derivative. As a result, the implementation of this method and its effectiveness and accuracy are presented explicitly.

From a physical aspect, the intrinsic nature of the physical system can be reflected to the mathematical model of the system by using fractional derivatives. Therefore, the solution of the fractional mathematical model is in excellent agreement with the predictions and experimental measurement of it. The systems whose behaviour is non-local can be modelled better by fractional mathematical models, and the degree of its non-locality can be arranged by the order of fractional derivative. In order to analyze the diffusion in a non-homogenous medium that has memory effects, it is better to analyze the solution of the fractional mathematical model for this diffusion. As a result, in order to model a process, the correct choices of fractional derivative and its order must be determined.

In the mathematical modelling of a diffusion problem for different matters such as liquid, gas and temperature, the suitable fractional order $\alpha$ is chosen, since the diffusion coefficient depends on the order $\alpha$ of fractional derivative [15]. This mathematical modelling describes the behaviour of matter in a phase. There is a vast amount of published work on the diffusion of various matters in science, especially in fluid mechanics and gas dynamics [16-19]. From this aspect, the analysis of this problem plays an important role in its application. Moreover, sub-diffusion cases for which $0<\alpha<1$ are under consideration. The solution of the fractional mathematical model of sub-diffusion cases behaves much slower than the solution of the integer-order mathematical model unlike the fractional mathematical model for super-diffusion [20]. 
The main goal of this study is to establish the analytic solution of following time fractional differential equations with the Dirichlet boundary and initial conditions.

$$
\begin{aligned}
{ }_{0}^{C P C} D_{t}^{\alpha} u(x, t) & =u_{x x}(x, t)+B u_{x}(x, t)-C u(x, t) \\
u(0, t) & =u(l, t)=0, \\
u(x, 0) & =f(x) e^{-\frac{B}{2} x}
\end{aligned}
$$

where $0<\alpha<1,0 \leq x \leq l, 0 \leq t \leq T, B, C \in \mathbb{R}$.

\section{Main results}

The analytic form of the solution for the problem (10)-(12) is established by employing the well known separation of variables method.

$$
u(x, t ; \alpha)=X(x) T(t ; \alpha)
$$

where $0 \leq x \leq l, 0 \leq t \leq T$.

Utilizing (13) in (10) and some arrangement leads to the following:

$$
\frac{{ }_{0}^{C P C} D_{t}^{\alpha}(T(t ; \alpha))}{T(t ; \alpha)}+C=\frac{X^{\prime \prime}(x)+B X^{\prime}(x)}{X(x)}=-\lambda .
$$

Taking the right hand side of equation (14) and related boundary conditions (11) into account, the following problem is obtained:

$$
\begin{array}{r}
X^{\prime \prime}(x)+B X^{\prime}(x)+\lambda X(x)=0, \\
X(0)=X(l)=0,
\end{array}
$$

which has the solution $X(x)=e^{r x}$. As a result, the following characteristic equation is reached $r^{2}+B r+\lambda=0$.

Case 1. If $B^{2}-4 \lambda>0$, the solutions of the characteristic equation are two distinct real roots $r_{1}, r_{2}$ which lead to the solution of the problem (15)-(16) as $X(x)=$ $c_{1} e^{r_{1} x}+c_{2} e^{r_{2} x}$. The first boundary condition yields $X(0)=0=c_{1}+c_{2}$ which leads to $c_{1}=-c_{2}$. Similarly, the second boundary condition leads to $X(l)=c_{1}\left(e^{r_{1} l}-e^{r_{2} l}\right)=$ $0 \Longrightarrow c_{1}=0, c_{2}=0$, which implies that there is not any solution for $B^{2}-4 \lambda>0$.

Case 2. If $B^{2}-4 \lambda=0$, the solutions of the characteristic equation are two coincident real roots $r_{1}=r_{2}$, which lead to the solution of the problem (15)-(16) as $X(x)=c_{1} e^{r_{1} x}+c_{1} e^{r_{2} x}$. By making use of the first boundary condition, we have $X(0)=c_{1}=0$. Similarly, the second boundary condition leads to $X(l)=c_{2} l e^{r_{1} l} \Longrightarrow$ $c_{2}=0$, which implies that there is no solution for $B^{2}-4 \lambda=0$.

Case 3. If $B^{2}-4 \lambda<0$, the solutions of the characteristic equation are two complex roots which lead to the solution of the problem (15)-(16) as 
$X(x)=e^{-\frac{B}{2} x}\left(k_{1} \cos \left(\frac{\sqrt{4 \lambda-B^{2}}}{2} x\right)+i k_{2} \sin \left(\frac{\sqrt{4 \lambda-B^{2}}}{2} x\right)\right)$.

By making use of the first boundary condition, we have $X(0)=k_{1}=0$ which implies that $\sin \left(\frac{\sqrt{4 \lambda-B^{2}}}{2} l\right)=0$ which yields the following eigenvalues

$$
\lambda_{n}=\frac{4 w_{n}^{2}+B^{2} l^{2}}{4 l^{2}}=\frac{4 w_{n}^{2}+(B l)^{2}}{(2 l)^{2}}, n=0,1,2,3, \ldots, \lambda_{1}<\lambda_{2}<\lambda_{3}<\ldots
$$

where $w_{n}=n \pi, n=0,1,2,3, \ldots$, satisfy the equation

$\sin \left(w_{n}\right)=\sin \left(\frac{\sqrt{4 \lambda_{n}-B^{2}}}{2} l\right)=0$.

As a result, the solution is obtained as follows:

$$
X_{n}(x)=\sin \left(w_{n}\left(\frac{x}{l}\right)\right) e^{-\frac{B}{2} x}, n=1,2,3, \ldots
$$

The second equation in (14) for eigenvalue $\lambda_{n}$ yields the ordinary differential equation below:

$$
\frac{{ }_{0}^{C P C} D_{t}^{\alpha}(T(t ; \alpha))}{T(t ; \alpha)}=-(C+\lambda)
$$

which yields the following solution [1]

$$
T_{n}(t ; \alpha)=E_{\alpha, 1,1}^{1}\left(\frac{-\left(C+\frac{4 w_{n}^{2}+(B l)^{2}}{(2 l)^{2}}\right)}{K_{0}(\alpha)} t^{\alpha}, \frac{-K_{1}(\alpha)}{K_{0}(\alpha)} t\right), n=0,1,2,3, \ldots
$$

where a bivariate Mittag-Leffler function $E_{\alpha, \beta, \kappa}^{(\gamma)}(x, y)$ proposed by Fernandez et al. [21], is represented in the double power series as follows:

$E_{\alpha, \beta, \kappa}^{(\gamma)}(x, y)=\sum_{r=0}^{\infty} \sum_{s=0}^{\infty} \frac{(\gamma)_{r+s}}{\Gamma(\alpha r+\beta s+\kappa)} \frac{x^{r}}{r !} \frac{y^{s}}{s !}, \alpha, \beta, \gamma \in \mathbb{C}, \operatorname{Re}(\alpha), \operatorname{Re}(\beta), \operatorname{Re}(\kappa)>0$

The solution for every eigenvalue $\lambda_{n}$ is constructed as

$u_{n}(x, t ; \alpha)=X_{n}(x) T_{n}(t ; \alpha)=E_{\alpha, 1,1}^{1}\left(\frac{-\left(C+\frac{4 w_{n}^{2}+(B l)^{2}}{(2 l)^{2}}\right)}{K_{0}(\alpha)} t^{\alpha}, \frac{-K_{1}(\alpha)}{K_{0}(\alpha)} t\right) \sin \left(w_{n}\left(\frac{x}{l}\right)\right) e^{-\frac{B}{2} x}, n=0,1,2,3, \ldots$ 
which leads to the following general solution

$$
u(x, t ; \alpha)=\sum_{n=1}^{\infty} A_{n} e^{-\frac{B}{2} x} \sin \left(w_{n}\left(\frac{x}{l}\right)\right) E_{\alpha, 1,1}^{1}\left(\frac{-\left(C+\frac{4 w_{n}^{2}+(B l)^{2}}{(2 l)^{2}}\right)}{K_{0}(\alpha)} t^{\alpha}, \frac{-K_{1}(\alpha)}{K_{0}(\alpha)} t\right) \text {. }
$$

The convergence of the series in (22) is proved in [1].

Note that it satisfies the boundary condition and fractional differential equation.

The coefficients of a general solution are established by taking the following initial condition into account:

$$
u(x, 0)=f(x) e^{-\frac{B}{2} x}=\sum_{n=1}^{\infty} A_{n} e^{-\frac{B}{2} x} \sin \left(w_{n}\left(\frac{x}{l}\right)\right) .
$$

The coefficients $A_{n}$ for $n=1,2,3, \ldots$ determined by the help of inner product with weighted function defined on $L^{2}[0, l]$ as $\langle u, v\rangle=\frac{2}{l} \int_{0}^{l} u(x) v(x) e^{B x} d x$ :

$A_{n}=\frac{2}{l}<e^{-\frac{B}{2} x} \sin \left(w_{n}\left(\frac{x}{l}\right)\right), f(x) e^{-\frac{B}{2} x}>=\frac{2}{l} \int_{0}^{l} \sin \left(w_{n}\left(\frac{x}{l}\right)\right) e^{-\frac{B}{2} x} f(x) e^{-\frac{B}{2} x} e^{B x} d x=\frac{2}{l} \int_{0}^{l} \sin \left(\frac{n \pi x}{l}\right) f(x) d x$

The advantage of this method comparing the homotopy method or other numerical methods is that exact solutions of the fractional differential equations are established by the separation of variables method, while their approximate solutions are acquired by homotopy and other numerical methods. Although the separation of variables method is a very common method to construct the solution of partial differential equations, applying it to fractional differential equations is not included in a large number of studies in literature.

\section{Illustrative example}

Let the following mathematical problem be considered:

$$
\begin{aligned}
& u_{t}=u_{x x}+u_{x}-u, \\
& u(0, t)=u(2, t)=0, \\
& u(x, 0)=-\sin (\pi x) e^{-\frac{1}{2} x}
\end{aligned}
$$

whose solution is given in the following form:

$$
u(x, t)=-e^{-\frac{1}{2} x} \sin (\pi x) e^{-\left(\pi^{2}+\frac{5}{4}\right) t}
$$


where $0 \leq x \leq 2,0 \leq t \leq T$.

Now the following time fractional form of the above problem is taken in hand:

$$
\begin{aligned}
{ }_{0}^{C P C} D_{t}^{\alpha} u(x, t) & =u_{x x}(x, t)+u_{x}(x, t)-u(x, t), \\
u(0, t) & =u(2, t)=0, \\
u(x, 0) & =\sin (\pi x) e^{-\frac{1}{2} x}
\end{aligned}
$$

where $0<\alpha<1,0 \leq x \leq 2,0 \leq t \leq T$.

The separation of variables method yields the following equations:

$$
\frac{{ }_{0}^{C P C} D_{t}^{\alpha}(T(t ; \alpha))}{T(t ; \alpha)}+1=\frac{X^{\prime \prime}(x)+X^{\prime}(x)}{X(x)}=-\lambda .
$$

Taking the right hand side of equation (30) and related boundary conditions (28) into account, the following problem is obtained:

$$
\begin{aligned}
X^{\prime \prime}(x)+X^{\prime}(x)+\lambda X(x) & =0, \\
X(0)=X(2) & =0,
\end{aligned}
$$

The representation of the solution for the eigenvalue problem (31)-(32) is obtained as

$$
X_{n}(x)=e^{-\frac{1}{2} x} \sin \left(\frac{n \pi x}{2}\right), n=1,2,3, \ldots
$$

The second equation in (30) for every eigenvalue $\lambda_{n}$ yields the following equation:

$$
\frac{{ }_{0}^{C P C} D_{t}^{\alpha}(T(t ; \alpha))}{T(t ; \alpha)}=-(1+\lambda)
$$

which has the following solution

$$
T_{n}(t ; \alpha)=E_{\alpha, 1,1}^{1}\left(\frac{-\left(1+\frac{n^{2} \pi^{2}+1}{4}\right)}{K_{0}(\alpha)} t^{\alpha}, \frac{-K_{1}(\alpha)}{K_{0}(\alpha)} t\right), n=0,1,2,3, \ldots
$$

For each eigenvalue $\lambda_{n}$, we obtain the following solution:

$$
u_{n}(x, t ; \alpha)=E_{\alpha, 1,1}^{1}\left(\frac{-\left(1+\frac{n^{2} \pi^{2}+1}{4}\right)}{K_{0}(\alpha)} t^{\alpha}, \frac{-K_{1}(\alpha)}{K_{0}(\alpha)} t\right) \sin \left(\frac{n \pi x}{2}\right), n=0,1,2,3, \ldots
$$

and hence the superposition principle leads to the following sum:

$$
u(x, t ; \alpha)=\sum_{n=1}^{\infty} A_{n} e^{-\frac{1}{2} x} \sin \left(\frac{n \pi x}{2}\right) E_{\alpha, 1,1}^{1}\left(\frac{-\left(1+\frac{n^{2} \pi^{2}+1}{4}\right)}{K_{0}(\alpha)} t^{\alpha}, \frac{-K_{1}(\alpha)}{K_{0}(\alpha)} t\right) .
$$


Utilizing the weighted inner product and initial condition (29) allows us to determine the coefficients $A_{n}$ as follows:

$$
-\sin (\pi x) e^{-\frac{1}{2} x}=\sum_{n=1}^{\infty} A_{n} e^{-\frac{1}{2} x} \sin \left(\frac{n \pi x}{2}\right) .
$$

The coefficients $A_{n}$ for $n=0,1,2,3, \ldots$ are determined by the help of the inner product as follows:

$A_{n}=<\sin \left(\frac{n \pi x}{2}\right) e^{-\frac{1}{2} x},-\sin (\pi x) e^{-\frac{1}{2} x}>=\int_{0}^{2} \sin \left(\frac{n \pi x}{2}\right) e^{-\frac{1}{2} x}(-\sin (\pi x)) e^{-\frac{1}{2} x} e^{x} d x$

For $n \neq 2, A_{n}=0 . n=2$ we get

$A_{2}=-\int_{0}^{2} \sin ^{2}(\pi x) d x=-\int_{0}^{2}\left(\frac{1}{2}-\frac{\cos (2 \pi x)}{2}\right) d x=\left.\left(\frac{x}{2}-\frac{\sin (2 \pi x)}{4}\right)\right|_{x=0} ^{x=2}=-1$.

Thus

$$
u(x, t ; \alpha)=-e^{-\frac{1}{2} x} \sin (\pi x) E_{\alpha, 1,1}^{1}\left(\frac{-\left(\pi^{2}+\frac{5}{4}\right)}{K_{0}(\alpha)} t^{\alpha}, \frac{-K_{1}(\alpha)}{K_{0}(\alpha)} t\right) .
$$

The accuracy of the obtained solution is checked by substituting $\alpha=1$ into (41) which leads to the solution of the problem (25).

Particularly, the solution of the problem (27)-(29) has the following form for the specific functions $K_{0}$ and $K_{1}$ :

Case 1: For $K_{0}(\alpha)=\alpha, K_{1}(\alpha)=1-\alpha$, the solution becomes

$$
u(x, t ; \alpha)=-e^{-\frac{1}{2} x} \sin (\pi x) E_{\alpha, 1,1}^{1}\left(\frac{-\left(\pi^{2}+\frac{5}{4}\right)}{\alpha} t^{\alpha}, \frac{\alpha-1}{\alpha} t\right) .
$$

Case 2: For $K_{0}(\alpha)=\alpha^{2}, K_{1}(\alpha)=1-\alpha^{2}$, the solution becomes

$$
u(x, t ; \alpha)=-e^{-\frac{1}{2} x} \sin (\pi x) E_{\alpha, 1,1}^{1}\left(\frac{-\left(\pi^{2}+\frac{5}{4}\right)}{\alpha^{2}} t^{\alpha}, \frac{\alpha^{2}-1}{\alpha^{2}} t\right) .
$$

The graphics of solutions for Case 1, Case 2 and Problem (25) in 2D are given in Figures 1a-d and Figures 2a-d are given in 3D for various values of $\alpha$.

Note that by truncated solution we mean the approximate solution.

It is clear from Figures 1a-d and 2a-d that the solution of time fractional diffusion equation including the constant proportional Caputo hybrid operator derivative ${ }_{0}^{C P C} D_{\alpha}{ }^{1} f(t)$ converges the solution of the integer order diffusion equation as $\alpha$ tends to 1 faster than its solution including the constant proportional Caputo hybrid operator derivative ${ }_{0}^{C P C} D_{\alpha}{ }^{2} f(t)$. As a result, we conclude that the choice of the functions 


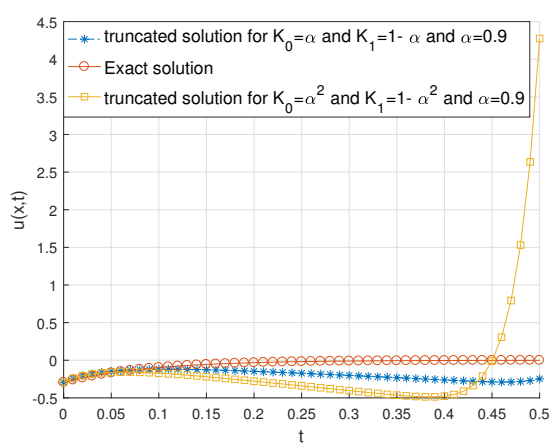

(a) $\alpha=0.9$

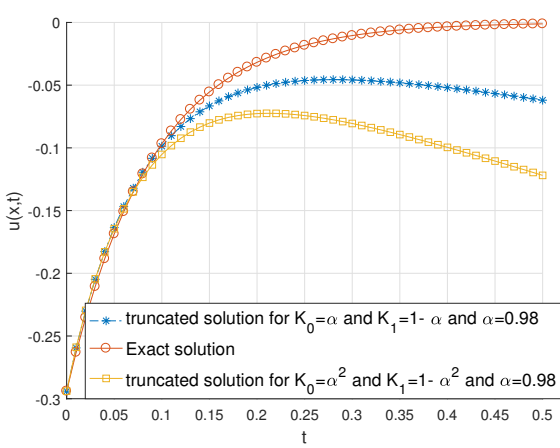

(c) $\alpha=0.98$

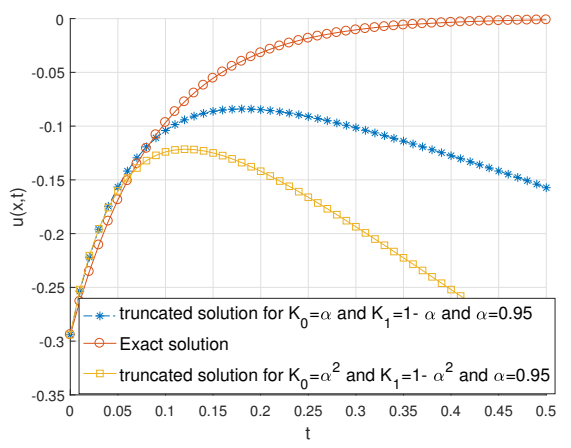

(b) $\alpha=0.95$

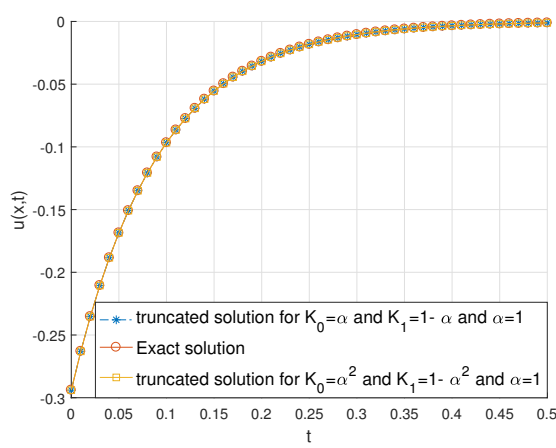

(d) $\alpha=1$

Fig. 1. The graphics of solutions for Example for different functions $K_{0}(\alpha)$ and $K_{1}(\alpha)$ in 2D at $x=0.1$ and for various values $\alpha$

$K_{0}(\alpha)$ and $K_{1}(\alpha)$ in ${ }_{0}^{C P C} D_{\alpha}{ }^{1} f(t)$ are better than those in ${ }_{0}^{C P C} D_{\alpha}{ }^{2} f(t)$. Moreover, the graphs of the solutions move away from the solution of the corresponding integer order differential equation, as the fractional order $\alpha$ decreases away from 1 .

\section{Conclusion}

The solution of the mathematical problem with the hybrid time fractional derivative is constructed by the separation of variables method in terms of the bivariate Mittag-Leffler function. Besides, the accuracy of the solution is tested by taking $\alpha=1$ in the solution which leads to the solution of the mathematical problem with ordinary derivative. As a result, the illustrative example indicates that the separation of variables method plays an influence role in the construction of mathematical problems including fractional derivatives.

Based on the analytic solution, we reach the conclusion that diffusion processes decay over time until an initial condition is reached when $\alpha$ is less than a certain value of $\alpha$ for Case 1 but diffusion processes decay with time for all values of $\alpha$ between 0 and 1 for Case 2. As $\alpha$ tends to 0 , the rate of decaying increases. This 


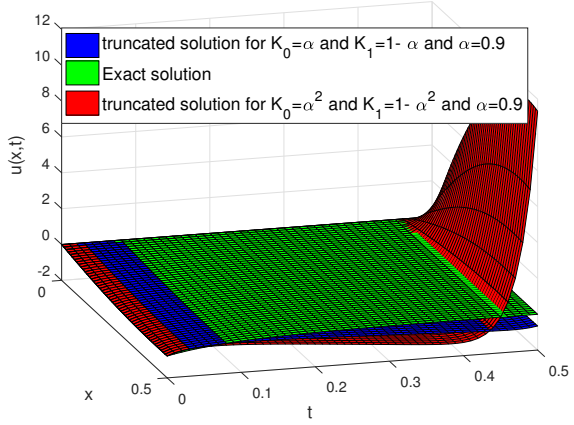

(a) $\alpha=0.9$

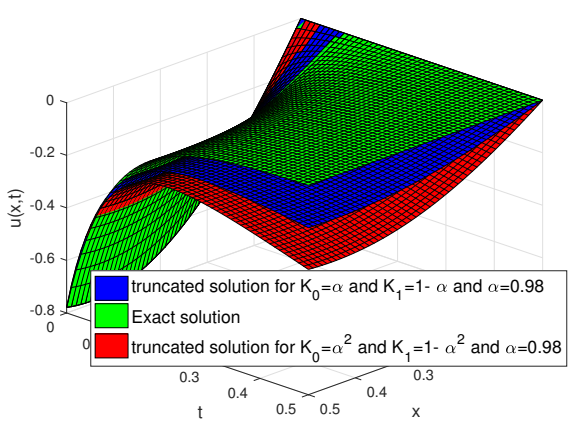

(c) $\alpha=0.98$

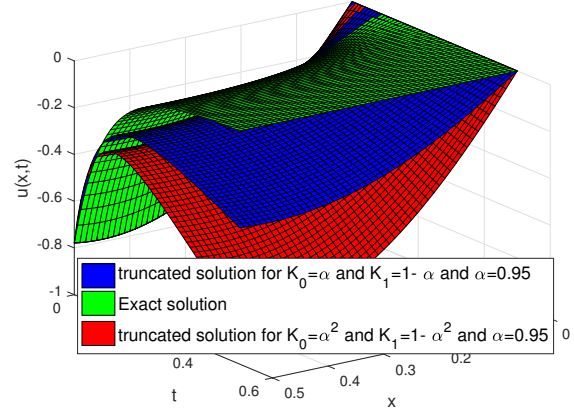

(b) $\alpha=0.95$

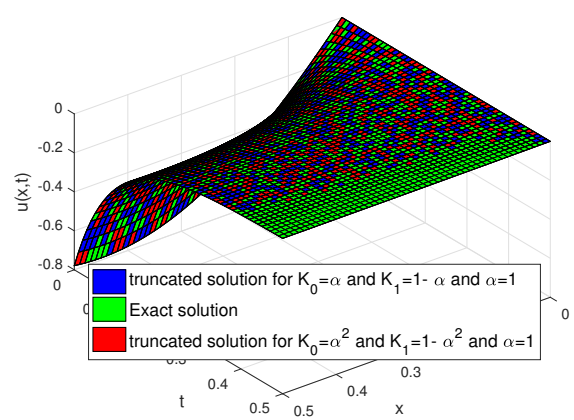

(d) $\alpha=1$

Fig. 2. The graphics of solutions for Example for different functions $K_{0}(\alpha)$ and $K_{1}(\alpha)$ in $3 \mathrm{D}$ for various values $\alpha$

implies that in the mathematical model for diffusion of the matter which has a small diffusion rate, the value of $\alpha$ must be close to 0 . This model can account for various diffusion processes of various methods.

\section{References}

[1] Baleanu, D., Fernandez, A., \& Akgul, A. (2020). On a fractional operator combining proportional and classical differintegrals. Mathematics, 8(360).

[2] Sweilam, N.H., Al-Mekhlafi, S.M., \& Baleanu, D. (2020). A hybrid fractional optimal control for a novel Coronavirus (2019-nCov) mathematical model. Journal of Advanced Research, DOI: $10.1016 /$ j.jare.2020.08.006.

[3] Sweilam, N.H., Al-Mekhlafi, S.M., Almutairi, A., \& Baleanu, D. (2021). A hybrid fractional COVID-19 model with general population mask use: Numerical treatments. Alexandria Engineering Journal, 60(3), 3219-3232.

[4] Ibrahim, R.W., \& Baleanu, D. (2021). On quantum hybrid fractional conformable differential and integral operators in a complex domain. Revista de la Real Academia de Ciencias Exactas, Físicas y Naturales. Serie A. Matemáticas, 115, 31, DOI: 10.1007/s13398-020-00982-5.

[5] Aslam, M., Murtaza, R., Khan, H., Baleanu, D., \& Khan, A. (2020). Singular hybrid fractional differential systems. Authorea, DOI: 10.22541/au.158480068.83814487. 
[6] Fernandez, A., Kürt, C., \& Özarslan, M.A. (2020). A naturally emerging bivariate Mittag-Leffler function and associated fractional-calculus operators. Computational and Applied Mathematics, 39, Article number: 200(2020).

[7] Asjad, M.I., Ikram, M.D., Ali, R., Baleanu, D., \& Alshomrani, A.S. (2020). New analytical solutions of heat transfer flow of clay-water base nanoparticles with the application of novel hybrid fractional derivative. Thermal Science, 24(Suppl. 1), 343-350.

[8] Chu, Y.M., Khan, U., Ahmed, N., Mohyud-Din, S.T., \& Khan, I. (2020). Heat and mass transport investigation in radiative and chemically reacting fluid over a differentially heated surface and internal heating. Open Physics, DOI: 10.1515/phys-2020-0182.

[9] Geng, F., \& Wu, X. (2021). Kernel functions-based approach for distributed order diffusion equations. Numerical Methods for Partial Differential Equations, 37, 1269-1281.

[10] Ikram, M.D., Asjad, M.I., Akgül, A., \& Baleanu, D. (2021). Effects of hybrid nanofluid on novel fractional model of heat transfer flow between two parallel plates. Alexandria Engineering Journal, 60(4), 3593-3604.

[11] Günerhan, H., Dutta, H. Dokuyucu, M.A., \& Adel, W. (2020). Analysis of a fractional HIV model with Caputo and constant proportional Caputo operators. Chaos, Solitons \& Fractals, 139,110053

[12] Acay, B., \& Inc, M. (2021). Fractional modeling of temperature dynamics of a building with singular kernels. Chaos, Solitons \& Fractals, 142, 110482.

[13] Asjad, M.I. (2020). Novel fractional differential operator and its application in fluid dynamics. Journal of Prime Research in Mathematics, 16(2), 67-79.

[14] Ahmad, M., Imran, M.A., Baleanu, D., \& Alshomrani, A.S. (2020). Thermal analysis of magnetohydrodynamic viscous fluid with innovative fractional derivative. Thermal Science, 24(Supp. 1), 351-359.

[15] Bisquert, J. (2005). Interpretation of a fractional diffusion equation with nonconserved probability density in terms of experimental systems with trapping or recombination. Physical Review E, 72, 011109.

[16] Cetinkaya, S., Demir, A., \& Kodal Sevindir, H. (2020). The analytic solution of initial boundary value problem including time-fractional diffusion equation. Facta Universitatis Ser. Math. Inform., 35(1), 243-252.

[17] Cetinkaya, S., \& Demir, A. (2019). The analytic solution of time-space fractional diffusion equation via new inner product with weighted function. Communications in Mathematics and Applications, 10(4), 865-873.

[18] Cetinkaya, S., \& Demir, A. (2020). Time fractional diffusion equation with periodic boundary conditions. Konuralp Journal of Mathematics, 8(2), 337-342.

[19] Cetinkaya, S., \& Demir, A. (2020). Time fractional equation including non-homogenous Dirichlet boundary conditions. Sakarya University Journal of Science, 24(6), 1185-1190.

[20] Naber, M. (2004). Distributed order fractional sub-diffusion. Fractals, 12(1), 23-32.

[21] Fernandez, A., Kurt, C., \& Ozarslan, M.A. (2020). A naturally emerging bivariate Mittag-Leffler function and associated fractional-calculus operators. Computational and Applied Mathematics, $39(200)$. 